Relations industrielles

Industrial Relations

\title{
Antécédents et efficacité des stratégies de médiation de conflits : le cas des présidents de Commissions mixtes paritaires en France
}

\section{Antecedents and Effectiveness of Mediation Strategies: The Intervention of Presidents of Joint Committees in France Antecedentes y eficacidad de las estrategias de mediación: la intervención de los presidentes de Comisiones mixtas paritarias en Francia}

\author{
François Grima, Olivier Brunel, Irène Georgescu et Ludovic Taphanel
}

Volume 73, numéro 3, été 2018

URI : https://id.erudit.org/iderudit/1053837ar

DOI : https://doi.org/10.7202/1053837ar

Aller au sommaire du numéro

Éditeur(s)

Département des relations industrielles de l’Université Lava

ISSN

0034-379X (imprimé)

1703-8138 (numérique)

Découvrir la revue

Citer cet article

Grima, F., Brunel, O., Georgescu, I. \& Taphanel, L. (2018). Antécédents et efficacité des stratégies de médiation de conflits : le cas des présidents de Commissions mixtes paritaires en France. Relations industrielles / Industrial Relations, 73(3), 461-485. https://doi.org/10.7202/1053837ar

\section{Résumé de l'article}

Le but de cet article est d'identifier les stratégies développées par le médiateur, ainsi que leurs antécédents. S'appuyant sur une enquête quantitative réalisée auprès de 51 présidents de Commission mixte paritaire (PCMP) ayant un rôle de médiateur au niveau des branches professionnelles, cette recherche établit deux résultats.

Premièrement, elle distingue deux stratégies développées par le médiateur : la stratégie d'accompagnement et la stratégie interventionniste. La première permet au médiateur d'amener les parties au conflit à l'accord. La seconde stratégie n'a pas d'effets directs sur la résolution de l'accord. Cependant, nos résultats démontrent l'existence d'un effet indirect de cette stratégie interventionniste. Celle-ci est efficace lorsqu'elle est associée à la stratégie d'accompagnement.

Deuxièmement, l'intervention d'un tiers expert ou d'autorité, soit un suivi de l'activité des parties en dehors de la salle de médiation, facilite la stratégie interventionniste alors que la compétence des parties, notamment un contact régulier avec ces dernières, est lié à la stratégie d'accompagnement. Dépassant les approches descriptives, ce travail propose une meilleure compréhension du comportement du médiateur, une intervention dynamique et complexe lors du processus de médiation.

Cette recherche apparait comme un nouveau point de départ pour comprendre les dynamiques de médiations dans un contexte où le rôle des partenaires sociaux dans la définition de la réglementation sociale s'affirme. Les auteurs suggèrent d'intégrer dans l'analyse des dynamiques de médiation, les facteurs contextuels et personnels liés aux différents acteurs que sont le médiateur, les parties prenantes, ainsi que les tiers extérieurs.

Les limites de ce travail demeurent la taille de son échantillon, ainsi que l'absence de triangulation des sources qui auraient permis d'obtenir une vision plus éclairée de l'action du médiateur. De plus, l'étude ayant été conduite en France, elle n'exclut pas l'existence de biais culturels. C'est pourquoi ce travail ouvre des perspectives multiples de recherches sur le sujet du rôle du médiateur dans les dynamiques de médiations.
Tous droits réservés @ Département des relations industrielles de l’Université Laval, 2018
Ce document est protégé par la loi sur le droit d'auteur. L’utilisation des services d'Érudit (y compris la reproduction) est assujettie à sa politique d'utilisation que vous pouvez consulter en ligne.

https://apropos.erudit.org/fr/usagers/politique-dutilisation/ 


\title{
Antécédents et efficacité des stratégies de médiation de conflits: le cas des présidents de Commissions mixtes paritaires en France
}

\author{
François Grima, Olivier Brunel, Irène Georgescu \\ et Ludovic Taphanel
}

\begin{abstract}
Le but de cet article est de comprendre la dynamique de médiation en associant ses antécédents, ses différentes manifestations et un résultat: l'accord. Théoriquement, en proposant une approche synthétique qui intègre des éléments contextuels, comme la présence de tiers ou les caractéristiques des médiateurs, cette recherche réalise une contribution académique importante, d'autant que ces éléments sont demeurés ignorés par la littérature. Empiriquement, le choix du contexte français est porteur d'enseignements managériaux importants. Lorsque le nouveau gouvernement redéfinit les fondements du droit du travail en donnant un nouveau rôle à la branche professionnelle, mieux comprendre comment l'action d'un médiateur valorise l'émergence d'un accord nous apparaît fructueux pour tous les partenaires. De même, ce travail permet de valoriser la complexité du rôle tenu par le médiateur pour l'obtention d'un accord.
\end{abstract}

MOTS-CLÉS: médiateur, médiation, stratégie, relations sociales.

\section{Introduction}

Définie comme « un processus de gestion des conflits où les parties (en conflit) recherchent ou acceptent une offre d'aide d'un individu, d'un groupe, d'un État ou d'une organisation pour régler leur conflit ou résoudre leurs différences sans recourir à la force physique ou invoquer l'autorité de la loi » (Bercovitch, Anagnoson et Wille, 1991 : 8), la médiation constitue une pratique de règlement alternatif des différends en plein développement, que cela soit dans le champ des relations internationales, personnelles, commerciales ou sociales (Wall et Dunne,

François Grima, professeur des Universités, Université Paris Est Créteil, France (Grimaf2000@yahoo.com). Olivier Brunel, maître de conférences, Université Lyon 3, Lyon, France (olbrunel@yahoo.fr).

Irène Georgescu, professeure des Universités, Université de Montpellier I, MOMA, Montpellier, France (Irene. georgescu@montpellier.fr).

Ludovic Taphanel, professeur assistant, Institut de Gestion sociale, École des ressources humaines,

Laboratoire Innovation sociale et de la Performance économique, Paris, France (Itaphanel@groupe-igs.fr). 
2012). Medina et al. (2014) expliquent cette évolution par le besoin de justice non satisfait par le système judiciaire. Cet intérêt a donné lieu à de multiples travaux académiques. Le cœur de la littérature sur la médiation se polarise sur les parties en conflit ou son contexte (Wall, Stark et Standifer, 2001), et la compréhension du travail du médiateur demeure une zone à explorer (Wall et Dunne, 2012). Le but de cette recherche est de contribuer à cette compréhension en se focalisant sur l'analyse des stratégies développées par le médiateur et leurs antécédents.

Pour atteindre ces objectifs, nous nous appuierons sur le cas des présidents des Commissions mixtes paritaires (PCMP) au sein des branches professionnelles en France. Ces commissions réunissent les partenaires sociaux syndicaux et patronaux, elles sont présidées par un représentant du ministre du Travail qui intervient en tant que médiateur pour faciliter le dialogue social. Plusieurs raisons expliquent ce choix. En France, la première est la dichotomie entre le développement de la pratique de la médiation dans le champ des relations sociales et l'absence de travaux académiques sur le sujet. Exceptés quelques travaux de portée générale décrivant l'essor de la médiation (Le Flanchec et Rojot, 2009; Stimec et Adijès, 2015), rares sont les travaux empiriques à l'aborder concrètement. Seuls les travaux de Grima $(2005,2006)$ font exception. S'inscrivant dans une approche exploratoire, l'auteur décrit l'existence de quatre styles stratégiques parmi les PCMP. Un réel besoin théorique de connaissances circonstanciées existe pour dépasser cette approche descriptive et proposer des analyses intégrant les spécificités culturelles propres à chaque pays en matière de médiation (Martinez-Pecino et al., 2008). La seconde est l'importance du rôle du PCMP dans les négociations collectives en France. Loin d'être une partie marginale de la médiation, les CMP constituent un dispositif-clé dans plus de 90 branches professionnelles permettant le maintien du dialogue social. Comprendre l'action des PCMP, c'est s'inscrire dans une dynamique sociétale de compréhension du dialogue social en France. Cette compréhension apparaît urgente comme le soulignent le Rapport parlementaire du 8 juin 2016 et les Ordonnances de la Loi du Travail du 22 septembre 2017.

Les apports de notre travail sont doubles. D'une part, nous montrons que les stratégies mobilisées par le médiateur sont interreliées et peuvent être utilisées simultanément. Ainsi, la stratégie d'accompagnement permet de déboucher sur un accord plus aisément que la stratégie interventionniste. D'autre part, nos résultats soulignent le poids de plusieurs antécédents (présence d'un tiers, activités en dehors de la salle de médiation, compétence des parties) dans le développement des stratégies mobilisées menant à un accord entre les parties. Après une revue de la littérature portant sur les stratégies de médiation, leur efficacité et leurs antécédents qui nous permettra de construire notre corps d'hypothèses, nous exposerons la méthodologie et détaillerons les résultats qui seront discutés dans une dernière partie. 


\section{Cadre théorique et hypothèses}

\section{Stratégies de médiation: une lecture bi ou tridimensionnelle?}

L'étude des tactiques mobilisées par le médiateur a connu une accélération notable au cours de la dernière décennie (Wall et Dunne, 2012). Cependant, cette profusion n'est pas source de réelles connaissances des choix du médiateur. Les tactiques dégagées sont souvent redondantes, ce qui limite l'apport analytique. En outre, un grand nombre de travaux n'ont pas de bases empiriques ou se veulent être prescriptifs sur les tactiques les plus pertinentes (Carnevale et Pruitt, 1992; Henderson, 1996).

Partant de ce constat, Wall et Dunne (2012) proposent de penser les stratégies développées par le médiateur en distinguant deux orientations. La première traduit sa volonté de s'introduire dans la médiation en tant que tierce partie prenante au conflit. Le médiateur cherche à intervenir dans les débats en se positionnant comme un de ses acteurs. La seconde, au contraire, I'amène à se penser comme un tiers à côté du conflit, aidant les parties à reprendre le chemin du dialogue. On retrouve cette taxinomie dans le travail de Kolb (1981). Cette dernière distingue le profil du négociateur (ou deal maker) de celui d'orchestrateur. Le premier perçoit que les parties sont inexpérimentées et dans l'impasse. II se sent alors la responsabilité de créer un accord acceptable par tous. II rassemble des informations sur les faits et se renseigne sur les priorités des parties puis, il tente de faire se rapprocher les positions. Pour l'orchestrateur, l'accord est inévitable: I'accord appartient aux parties qui doivent le créer. II fait converger les parties vers des propositions de plus en plus proches. Pour cela, il laisse du temps et de l'espace pour voir les problèmes et leurs priorités. L'orchestrateur n'est jamais dans l'intervention. Cette typologie se retrouve partiellement dans les travaux de Kressel et Pruitt $(1985,1989)$ qui distinguent trois stratégies: contextuelle, réflexive et substantive. Avec la stratégie contextuelle, le médiateur facilite la résolution du conflit en agissant sur le contexte de la médiation et en rendant le contexte de la rencontre entre les parties le plus efficace possible. Avec la stratégie réflexive, le médiateur tisse des liens avec les parties pour créer une dynamique de confiance favorisant l'émergence d'un accord. Enfin, avec la stratégie substantive, le médiateur se positionne comme un acteur de la médiation au cœur du processus de résolution. Ici, le médiateur n'est plus neutre, il cherche à faire évoluer la position d'une des parties qui bloque, selon lui, la dynamique de la médiation. D'après Kressel et Pruitt (1989), quatre grandes orientations sont à distinguer : 1-établir un climat de confiance avec les parties; 2-améliorer le climat de confiance entre les parties; 3-faire émerger les problèmes; et 4-faire pression pour obtenir un accord. Pour sa part, Shapiro, Drieghe et Brett(1985) distinguent quatre profils: 1-le négociateur (deal maker), 2-les rondes diplomatiques (shuttle 
dip/omacy), 3-la mise sous pression de la partie patronale; et/ou 4-celle dela partie salariale.

Face à ce débat théorique autour de la dimensionnalité des stratégies développées par le médiateur, nous avons choisi de retenir une approche bidimensionnelle pour deux motifs: 1- le seul travail académique empirique réalisé sur le sujet (Grima, 2005) suggère une articulation entre interventionnisme et accompagnement. Si la stratégie substantive est clairement identifiable, en revanche les stratégies contextuelles et réflexives paraissent se combiner. Nous retrouvons les orientations de la formation proposée par le Ministère du Travail qui souligne l'articulation entre interventionnisme et accompagnement en mettant en avant la seconde; 2- Kolb (1981), tout comme Wall et Dunne (2012), concluent que la distinction entre intervention et accompagnement apparaît à la fois comme l'approche la plus stable empiriquement et théoriquement. Nous distinguerons donc la stratégie interventionniste traduisant la volonté du médiateur d'être une troisième partie de la médiation de la stratégie d'accompagnement (contextuelle et réflexive) où le médiateur facilite le dialogue en demeurant extérieur.

\section{L'efficacité des stratégies du médiateur}

La médiation est décrite par la littérature comme un procédé efficace de résolution des conflits. Dans les travaux de recherches, différents taux globaux de succès de la médiation sont observés : $60 \%$ pour Kressel et Pruitt (1989), $75 \%$ pour Wall, Stark et Stadinfer (2001), 80\% pour Wall et Dunne (2012). Également, des divergences selon le type de médiation existent (Wall et Dunne, 2012). Rares sont les recherches qui évaluent l'efficacité des différentes orientations stratégiques du médiateur (Martinez-Pecino et al., 2008). Le poids donné au contexte vient relativiser l'importance des orientations stratégiques. Quelles que soient les stratégies d'accompagnement, contextuelles ou réflexives, elles apparaissent comme ayant un impact positif sur la réalisation d'un accord entre les parties (Carnavale et Pruitt, 1992; Zartman et Touval, 1985). L'accompagnement permet aux parties d'optimiser leur compréhension réciproque dans des délais réduits. Le rôle de la confiance est également majeur dans la médiation (Wall et Lynn, 1993). Pour Hiltrop (1985), l'efficacité des médiateurs est optimale lorsque ces derniers agissent en tant que facilitateur. La confiance attribuée, manifestée aux parties, les conduit à leur tour à se montrer plus ouvertes aux positions des acteurs. Cependant, la stratégie d'accompagnement est tributaire du contexte conflictuel dans lequel s'insère la médiation (Martinez-Pecino et al., 2008) : plus il est tendu, plus elle peut s'avérer difficile et inefficace tant les parties ne sont pas confiantes dans la possibilité même d'un accord. À l'inverse, si le médiateur dispose, dès le démarrage, d'un mandat explicite pour agir, la stratégie d'accompagnement retrouve son efficacité. Sur ces bases, nous avançons I'hypothèse suivante: 
HYPOTHĖSE 1 La stratégie d'accompagnement a un impact positif direct sur la signature d'un accord pour les parties impliquées

Pour la stratégie interventionniste, la littérature est moins claire que pour I'accompagnement. Dans un contexte de médiation du travail, Posthuma, Dworkin et Swift (2002) souligne son efficacité dans un climat tendu. Un médiateur percutant, orientant la médiation sans laisser de place aux choix des parties, constitue un atout pour aller vers I'accord (Posthuma, 2000). Ainsi, la stratégie interventionniste semble avoir un effet positif direct sur la signature d'un accord. Ce résultat est cohérent avec les travaux de Kochan et Jick (1978). Pour Kolb (1994), dans une version idéalisée de la médiation, I'accord serait établi en dehors de toute intervention du médiateur. Or, la réalité des pratiques organisationnelles est différente et le médiateur se doit d'intervenir dans une médiation (Rothman, 2014), mais cette intervention doit être réfléchie. Cependant, ces résultats méritent d'être nuancés pour deux motifs. D'une part, ils ne doivent pas occulter la possibilité de résistance des parties. Une partie confrontée à un médiateur ne lui renvoyant pas une image positive de ses positions peut se radicaliser (Wall et Lynn, 1993). Cette grille de lecture est validée dans les contextes où la conflictualité n'est pas à un niveau de crise, comme cela est le cas dans les CMP. D'autre part, les partenaires sociaux sont réticents à l'égard de toute intervention étatique dans le dialogue social au niveau des branches (Grima, 2005). Le statut de fonctionnaire de 67 des 68 PCMP peut contribuer à renforcer cette perception d'intrusion.

Ces résultats suggèrent que les liens entre stratégie interventionniste et la signature d'un accord sont plus complexes. Elle semble agir directement mais, aussi, comme une première étape dans un processus d'influence plus élaboré. Conscient des différences dans les positions initiales, le médiateur aborderait la médiation avec une stratégie interventionniste pour les faire évoluer avant de développer une stratégie d'accompagnement une fois la dynamique de rapprochement initié. Wang (2015) souligne que, dans le contexte chinois, les médiateurs dans les relations de travail articulent les deux orientations. Ces différents éléments nous conduisent à poser les deux hypothèses suivantes:

HYPOTHÈSE 2 Pour les parties impliquées, la stratégie interventionniste a un impact positif direct sur la signature d'un accord.

HYPOTHĖSE 3 Pour les parties impliquées, la stratégie interventionniste a un impact positif indirect par l'intermédiaire de la stratégie d'accompagnement sur la signature d'un accord.

\section{Les antécédents des stratégies du médiateur}

Quelle que soit la revue de littérature de référence réalisée (Carnevale et Pruitt, 1992; Henderson, 1996; Wall, Stark et Standifer, 2001; Wall et Dunne, 2012), 
un consensus émerge sur le fait que la compréhension des antécédents des choix stratégiques demeure une zone d'ombre du champ. Ce sujet est un axe prioritaire de développement (Wall et Dunne, 2012). Selon Wall et Blum (1991) et Wall et Lynn (1993), plusieurs ensembles jouent un rôle dans ces choix: les facteurs liés à la personne du médiateur et les facteurs liés à son environnement d'action.

\section{Les facteurs liés à la personne du médiateur: la formation à la médiation}

La formation semble jouer un rôle-clé dans les orientations du médiateur. Quelle que soit les contextes (Girdner, 1986; McGillicuddy et al., 1987, Wall et Blum, 1991), la formation suivie pour les médiateurs orientent leurs choix stratégiques. Une formation orientée vers l'accompagnement plutôt que vers l'interventionnisme conduit à construire des médiateurs favorisant la première (McGillicuddy, Welton et Pruitt, 1987). L'existence d'une formation facultative aux fonctions de PCMP proposée par le Ministère du Travail valorisant une compréhension de facilitation du dialogue social, et d'accompagnement, nous conduit à penser que les PCMP I'ayant suivi seront plus tentés de valoriser la stratégie de facilitation par rapport à celle d'intervention. C'est pourquoi nous proposons I'hypothèse suivante:

HYPOTHĖSE 4 La formation suivie par le PCMP impacte positivement la mise en œuvre d'une stratégie d'accompagnement.

\section{Les facteurs contextuels: la compétence des parties et la présence d'un tiers lors de la médiation}

Au-delà du niveau de conflictualité entre les parties au moment de son intervention, ou de la pression temporelle dans laquelle s'inscrit son action, le médiateur doit prendre en considération les caractéristiques des parties. Le médiateur doit, aussi, tenir compte de l'expérience des parties à la médiation, notamment s'il existe un déficit de compétences Wall, Stark et Standifer (2001). Dans un contexte de dissymétrie de compétences entre les parties, le médiateur a tendance à opter pour la stratégie d'accompagnement à travers l'optimisation des agendas, la suggestion de voies de progrès, ne se posant pas comme un tiers incontournable (Wall et Lynn, 1993; Grima, 2005). Ce dernier choix risquerait de rigidifier la partie disposant de plus d'expertise, voyant alors le médiateur comme un allié de l'autre partie. Nous proposons I'hypothèse suivante:

HYPOTHÈSE 5 Le manque de compétence des parties a un impact positif sur la mise en œuvre d'une stratégie d'accompagnement.

La présence de tiers constitue une réalité mentionnée fréquemment dans les récits de médiateur (Wall et Dunne, 2012) alors, qu'à notre connaissance, peu de travaux académiques ne mentionnent cette variable (Wall, Stark et Standifer, 2001). Dans le cas des PCMP, deux types de tiers peuvent être introduits. 
Le premier est un expert qui vient éclairer la commission sur un point technique. Le second est un membre de l'administration centrale dont dépend la branche professionnelle, ce qui témoigne de l'intérêt porté par l'État au dialogue social dans la branche. Cette présence pèse sur les débats car elle représente l'autorité de l'État susceptible d'intervenir dans la branche professionnelle au cas où les accords trouvés ou la dynamique d'accord ne lui conviennent pas. Dans les deux cas, aucun de ces tiers n'a de pouvoir de décision et les débats demeurent sous le contrôle des parties et du PCMP. Pour autant, la littérature ne conçoit pas la mobilisation de tiers comme un moyen facilitant la mise sous pression des parties. L'introduction d'un tiers vise à faire prendre conscience aux parties des enjeux qu'elles ne voient pas (Bonta, 1996). De même, I'introduction d'un tiers constitue aussi un moyen pour le médiateur de rendre public la situation de médiation en révélant les faiblesses des parties, ce qui peut les faire évoluer (Pinkley et al., 1995). Ainsi, I'introduction d'un tiers peut constituer un élément amont d'une stratégie interventionniste.

C'est pourquoi nous posons la double hypothèse suivante:

HYPOTHÈSES L'introduction d'un tiers expert (hypothèse 6a) et d'un tiers d'autorité (hypothèse 6b) ont un impact positif sur la mise en œuvre d'une stratégie interventionniste.

\section{L'activité en dehors de la salle de médiation}

Les analyses sur le travail mené par le médiateur se concentrent sur ses activités dans la salle de médiation (Wall et Dunne, 2012). Or, le médiateur agit aussi en dehors. C'est ce que suggère la réflexion tant sur les stratégies contextuelles centrées sur l'amélioration de la relation entre les parties, que celles interventionnistes pour le cas des situations de pression temporelle (Ross et Wieland, 1996). En outre, la médiation s'insère dans un cadre légal, ce qui suppose de respecter un certain formalisme qui s'étend au-delà de la salle de médiation (Susskind, 1981; Tyler, 1987; Martinez-Pecino et al., 2008). Premièrement, un travail amont de compréhension de la situation, de la position des parties, de leurs enjeux peut être nécessaire (Barsky, 1983; Raiffa, 1983), travail qui peut avoir un impact sur le comportement des parties (Maoz et Terris, 2006; Wall et Chan-Serafin, 2009). Selon la stratégie du caucus (Welton et al., 1992; Poitras, 2013), il s'agit pour le médiateur de rentrer en contact avec les représentants de chaque partie, comme avec leurs supérieurs hiérarchiques, et d'animer des réunions avec les parties en dehors de la médiation. Ce premier axe peut être rapproché de la stratégie interventionniste, tant il témoigne d'une volonté amont de s'imposer auprès des parties comme un tiers négociateur au cœur de la médiation. Deuxièmement, les travaux de suivi et de collecte d'informations contribuent eux-aussi à la construction d'un accord. À l'inverse, ce second ensemble apparaît être un antécédent de 
la stratégie d'accompagnement. Sur cette base, nous proposons les deux hypothèses suivantes:

HYPOTHĖSE 7a Le contact avec les parties est lié positivement avec la stratégie interventionniste.

HYPOTHĖSE 7b Le suivi de la procédure est lié positivement avec la stratégie d'accompagnement.

Le modèle de cette recherche peut ainsi être représenté (cf. Figure 1).

FIGURE 1

Modèle de recherche

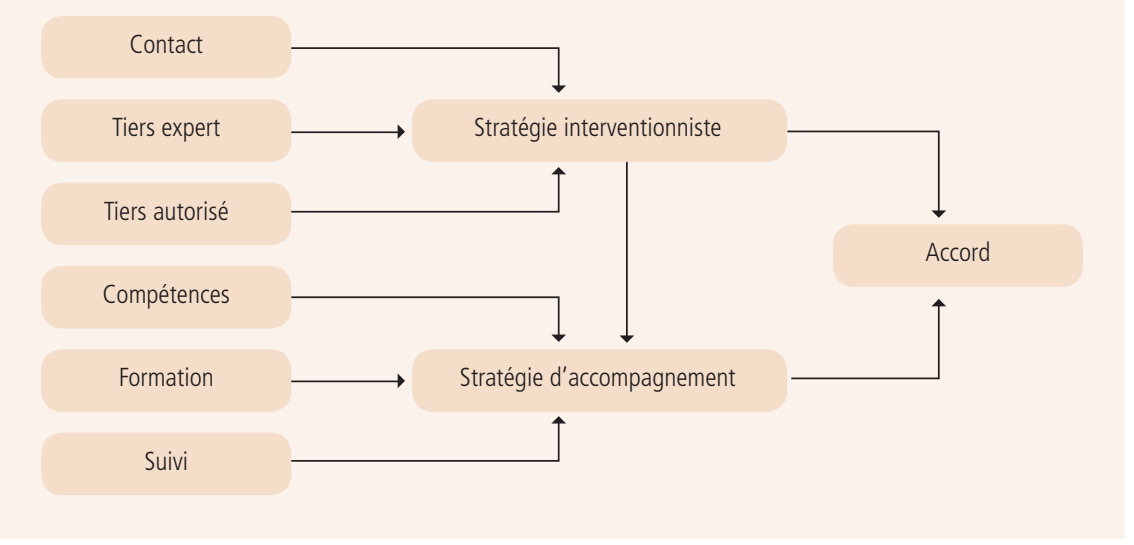

\section{Méthode de recherche}

\section{Contexte de la recherche}

Selon le Rapport parlementaire de Richard et Germain (2016), le paritarisme en France se caractérise par une négociation bilatérale. Cependant, l'État peut être sollicité par les partenaires sociaux pour contribuer à l'engagement et à l'avancement des négociations professionnelles. Cette mission se matérialise au niveau des branches professionnelles par la constitution de commissions mixtes paritaires (CMP). Monsieur Maurin, les définit ainsi : «l'objet d'une commission mixte paritaire [était] d'aider à la négociation de textes conventionnels de branche susceptibles d'extension, un tiers apportant une compétence technique et juridique pour mettre fin à des situations de blocage parfois passagères » (Cité, audition du 11 février 2016). Cette CMP se compose des représentants des organisations professionnelles d'employeurs et syndicales de salariés représentatives dans le secteur d'activité considéré et présidée par un représentant du ministre en charge du Travail. Ces commissions sont essentiellement à l'initiative des partenaires 
sociaux. Ici, l'État est présent de droit. Dans le cas de blocages persistants, la médiation peut provenir d'une initiative de l'État (article L. 2261-20 du Code du Travail). Plusieurs cas apparaissent: faible expérience de la négociation des parties, blocages et conflit intra-partie. Les CMP ne sont donc pas présentes dans toutes les branches professionnelles.

Pour sa part, Madame Rivoal, PCMP, précise que 94 des 300 branches professionnelles nationales étaient en CMP. 94 commissions sont animées par 68 PCMP dont 40\% sont des femmes. (Richard et Germain, 2016:105). Plus des trois quarts sont originaires du corps de l'inspection du travail, le reste étant constitué de fonctionnaires du Ministère du Travail (Richard et Germain, 2016: 105). Les tâches du PCM nous permettent de l'identifier comme un conciliateur (Grima, 2005). Le rapport parlementaire complète le propos en soulignant que le PCMP apporte: «aux partenaires sociaux un appui technique ou une expertise juridique» (104).

En outre, selon Monsieur Geiger :

Le PCMP n'intervient pas sur le contenu de leur négociation (celles des parties). II se pose modestement en facilitateur de leurs relations. II orchestre les prises de parole... le calendrier annuel de leurs rencontres, leur propose de hiérarchiser les thèmes de négociation, veille à la bonne tenue des réunions, etc. II suggère des approches et méthodes de travail permettant de faciliter les écoutes et compréhensions mutuelles et soutient leur volonté commune à construire la norme sociale de la branche. (Bilan de la négociation collective 2016: 235).

De plus, le Ministère du Travail définit le rôle du PCMP comme un conciliateur, un facilitateur'. Sa mission doit s'articuler autour de trois rôles: 1- un rôle d'animateur; 2- un rôle d'expert ou de conseil; 3- un rôle d'acteur où il se doit d'initier des propositions permettant de faire avancer les négociations. Ces différents éléments attestent de la volonté du Ministère du Travail de décrire le PCMP comme un conciliateur. On retrouve ici les huit «valeurs du conciliateur» décrites dans le Guide $d u$ conciliateur:1-la négociation est un mode efficace de règlement des problèmes de relations du travail; 2-les parties sont responsables du contenu de la négociation; 3-le conciliateur est au service des deux parties; 4-la convergence des intérêts est la voie privilégiée d'une entente satisfaisante pour les deux parties; 5-le conciliateur n'est pas un tiers décideurs et il sait travailler dans l'ombre; 6-le respect des personnes et du rôle de conciliateur constitue la base d'une conciliation efficace; 7-le conciliateur tend vers I'impartialité; et, finalement, 8-le conciliateur est ouvert à tous les possibles (Leblay, 2003). Cette description prescrite du rôle du PCMP comme conciliateur se retrouve partiellement chez Grima (2005) qui souligne que les PCMP se perçoivent comme des facilitateurs du dialogue social.

Cependant, les PCMP s'affirment aussi comme des acteurs soucieux de faire sortir la Commission de l'enlisement. Ils se perçoivent donc comme des acteurs 
de la négociation susceptibles d'élargir le débat, par la présence de tiers, par exemple. Ce positionnement du PCMP à la fois se rapproche et se distingue de celui de I'Inspecteur du Travail dans la résolution des conflits collectifs. L'article $R$ 2522-1 du Code du Travail encadre le rôle de l'Inspecteur du Travail qui: "assure un rôle de conseil et de conciliation en vue de la prévention et du règlement des conflits». Dans les faits, les Inspecteurs du Travail constituent les principaux interlocuteurs vers lesquels se tournent les parties en cas de conflits collectifs du travail du fait de leur compétence technique, de l'absence de formalisme de la démarche et de leur gratuité.

Si les deux interventions divergent sur la posture (formelle dans le cas du PCMP et informelle pour l'Inspecteur du Travail), il y a une volonté institutionnelle portée par le Ministère du Travail de décrire ces acteurs comme des conciliateurs visant «à réunir ensemble les parties dans des circonstances et une ambiance les plus propices à une discussion sereine pour la recherche d'un accord » (Touzard, 1977: 87). Le rôle de médiateur «englobe le précédent (conciliateur) mais ajoute une part plus active prise par le médiateur: il peut intervenir dans la discussion, faire des suggestions et des propositions ou même formuler des recommandations en vue d'un accord» (Ibid.: 87).

Sur cette base, il apparaît un hiatus entre une volonté institutionnelle d'assimilation du PCMP à un conciliateur et, de l'autre, des travaux empiriques qui suggèrent un positionnement possiblement plus actif. C'est pourquoi nous avons choisi de retenir le terme de médiateur pour traduire le fait que le PCMP n'a pas seulement pour rôle d'être un facilitateur, mais aussi d'être un acteur de la négociation. Notons que la distinction entre médiation et conciliation est minime tant: "les deux fonctions ont pour objectif d'organiser et de faciliter la négociation et la recherche de l'accord entre parties décidant en toute responsabilité et gardant le pouvoir final de décision pour elles-mêmes. » (Salzer et Vouche, 2003: 157).

\section{Échantillon}

Le questionnaire a été administré à l'ensemble de la population des PCMP français. Cette population est composée de 68 personnes. Le taux de retour a été de $92 \%$, ce qui nous a permis de collecter 63 questionnaires. Sur ce total, de nombreuses non réponses nous ont incités à exclure 12 répondants de notre échantillon. Au final, l'échantillon comprenait 51 négociateurs, ce qui représente $75 \%$ de la population mère. L'échantillon final comprenait 39\% de femmes et $61 \%$ d'hommes, et l'âge moyen des répondants était de 49 ans, avec un écarttype de 9,9 ans. L'ancienneté dans la fonction moyenne était de 6,65 années, avec un écart-type de 5,8 années. L'échantillon obtenu est représentatif de la population mère sur ces différentes caractéristiques. 


\section{Opérationnalisation des variables}

Le questionnaire est composé de 7 échelles. Le premier ensemble regroupe les échelles issues de la littérature (Lim et Carnevale, 1990) sur les stratégies de médiation, ses résultats et les raisons de son émergence. L'absence de recherche quantitative sur le terrain spécifique de médiation au niveau des branches professionnelles, et plus globalement au niveau de la thématique en France, comme la non traduction en français, à notre connaissance, de ces échelles, nous ont conduit à procéder à un renforcement de sa validité de contenu. Pour les trois variables, soit 1- stratégies de médiation; 2- accord; et 3-compétences des parties, nous avons conduit deux pré-enquêtes par technique d'entretien. Nous avons soumis les trois échelles à trois experts (anciens PCMP) qui ont évalué leur pertinence par rapport au contexte français de la négociation des branches en paritaires mixtes. Au terme de ces échanges, une sélection d'items a été retenue pour chacune des trois variables. Pour la variable stratégie de médiation nous avons retenus 15 items (ex: "J'ai développé des rapports amicaux avec les parties»), 7 items pour l'accord (ex: "Les désaccords sont résolus») et 4 items pour les compétences des parties (ex: "La conciliation s'explique par le déséquilibre de compétences techniques»). Ces échelles furent soumises à 10 PCMP afin de vérifier la bonne compréhension des items mobilisés. Ces échelles ont été mesurées sur la base d'une échelle de Likert à 5 points allant du «pas du tout d'accord » à «tout à fait d'accord» ou de «jamais» à «souvent», selon les variables.

Un second groupe de question repose sur des échelles que nous avons créées: 1 - activité en dehors de la salle de médiation; 2- intervention d'un tiers expert et d'autorité; et 3- la formation. Sur la base du travail de Grima (2005), nous avons conduit 5 entretiens avec des PCMP sur leur travail en dehors des séances de travail des CMP. Ces derniers ont permis d'extraire un ensemble de verbatim qui a été mobilisé afin d'élaborer les échelles de mesure. Nous avons, ensuite, procédé à l'évaluation de la validité du contenu. Nous avons tout d'abord soumis ces échelles à trois experts, puis nous avons pré-testé ces échelles auprès d'un échantillon de 10 PCMP (Roussel, 2005). Nous avons arrêté les entretiens à 10 personnes, ayant observé un effet de saturation. Le travail en dehors de la salle de médiation a été mesuré par une échelle de 10 items (par exemple: "Je rencontre les représentants des parties») mesurée par une échelle de Likert à cing points allant de 1 «jamais» à 5 "souvent». Pour l'échelle intervention de tiers (expert et autorité), nous avons procédé à la même démarche d'évaluation des contenus, ce qui nous a conduits à une échelle constituée de deux items. Le premier item définit la présence d'un «tiers autorité», à savoir: "un représentant d'une administration de tutelle est-il présent en tant qu'observateur lors des plénières? » et le second mesure le 
recours à un «tiers expert», soit: «Un expert sollicité par la commission est-il intervenu lors d'une plénière?». Nous avons mesuré la formation grâce à une échelle de 4 items (exemple: "La formation suivie sur le rôle des PCM a influencé ma pratique de PCM»).

\section{Traitements statistiques opérés}

Afin de tester les échelles, nous avons suivi le paradigme de Churchill (1979). Sur la base des pratiques observées (Igalens et Roussel, 1998), la taille de l'échantillon doit réunir cinq fois plus d'individus qu'il n'y a d'items soumis à une même analyse factorielle. Ces prescriptions normatives peuvent ne pas être suivies lorsque le test porte sur une population homogène et peu nombreuse (Roussel, 2005), comme dans notre cas. Les analyses ont été réalisées avec le logiciel SPSS 15.0 .

Au préalable, nous nous sommes assurés que les données sont «factorisables» (tests de Bartlett et indice KMO). Pour le test de dimensionnalité des différentes variables du modèle, nous avons réalisé des analyses en composantes principales (ACP). En ce qui concerne le nombre d'axes, nous avons retenu la restitution minimum de variance par la solution factorielle, le critère des valeurs propres et le «Scree Test de Cattell ». Au cours de la première itération, les échelles ont été soumises à l'analyse factorielle et au test de fiabilité $(\alpha$ de Cronbach). Les items ayant des contributions factorielles supérieures à 0,30 sur plusieurs facteurs ou n'ayant aucune contribution atteignant ce seuil sur un des facteurs retenus, tout comme ceux n'ayant pas de contribution supérieure ou égale à 0,6 sur l'une des composantes principales identifiées ont été éliminés. L'examen des communalités (au cours duquel nous avons supprimé les items dont la communalité est inférieure à 0,4$)$ a complété cette étape. Lors d'une seconde itération, les échelles modifiées ont fait l'objet d'un traitement statistique dont les résultats sont présentés dans le Tableau 1.

Les deux dimensions extraites par I'ACP laissent apparaître deux facteurs qui permettent d'envisager, conformément à Grima (2005), deux orientations de médiation: I'interventionnisme et l'accompagnement. Les qualités psychométriques des différentes mesures sont satisfaisantes pour l'ensemble des variables du modèle, les contributions factorielles des différents items sont proches ou supérieures au seuil de 0,7. La fiabilité des différentes mesures est également méritoire. Concernant l'activité des PCMP en dehors de la salle de médiation, deux dimensions sont apparues: 1 - le suivi et 2- le contact. Leurs qualités psychométriques (alphas, contributions factorielles et communalités) sont acceptables. Nous avons, ensuite, calculé un score (moyennes arithmétique) pour chacune des variables latentes que nous avons utilisées pour réaliser les régressions. Lorsque la variable n'était mesurée que par un item, nous avons utilisé le score de l'item. 
TABLEAU 1

Analyse en composantes principales des variables du modèle

\begin{tabular}{|c|c|c|c|c|}
\hline Facteur & $\alpha$ & $\begin{array}{l}\text { Valeur } \\
\text { propre }\end{array}$ & Item & $\lambda$ \\
\hline Suivi & 0,710 & 1,460 & $\begin{array}{l}\text { J'entre en lien avec la DGT pour suivre } \\
\text { l'évolution de la procédure d'extension une } \\
\text { fois l'accord déposé de ma propre initiative }\end{array}$ & 0,856 \\
\hline
\end{tabular}

J'entre en lien avec la DGT pour suivre l'évolution de la procédure d'extension une fois l'accord déposé à la demande des parties

Je me documente sur la réalité du travail dans la branche que j'anime

(Rapport de branche)

\begin{tabular}{|c|c|c|c|c|c|}
\hline \multirow[t]{2}{*}{ Compétences } & \multirow[t]{2}{*}{0,694} & \multirow[t]{2}{*}{1,217} & $\begin{array}{l}\text { La conciliation s'explique par un manque } \\
\text { d'autorité d'un des leaders des parties }\end{array}$ & 0,846 & 0,753 \\
\hline & & & $\begin{array}{l}\text { La conciliation s'explique par le déséquilibre } \\
\text { de compétences techniques }\end{array}$ & 0,880 & 0,781 \\
\hline \multirow[t]{3}{*}{ Accord } & \multirow[t]{3}{*}{0,734} & \multirow[t]{3}{*}{1,402} & Les désaccords sont résolus & 0,729 & 0,531 \\
\hline & & & $\begin{array}{l}\text { Un accord a été établi à la satisfaction de } \\
\text { l'ensemble ou d'une majorité des parties }\end{array}$ & 0,856 & 0,737 \\
\hline & & & $\begin{array}{l}\text { On peut penser qu'un accord est possible } \\
\text { sous peu }\end{array}$ & 0,695 & 0,600 \\
\hline \multirow[t]{4}{*}{ Contact } & \multirow[t]{4}{*}{0,701} & \multirow[t]{4}{*}{2,653} & Je rencontre les représentants des parties & 0,735 & 0,590 \\
\hline & & & $\begin{array}{l}\text { Je rencontre les supérieurs hiérarchiques } \\
\text { des parties }\end{array}$ & 0,728 & 0,645 \\
\hline & & & $\begin{array}{l}\text { Je téléphone aux représentants des parties } \\
\text { pour me tenir au courant de l'évolution } \\
\text { de leurs positions }\end{array}$ & 0,695 & 0,643 \\
\hline & & & $\begin{array}{l}\text { J'anime occasionnellement des groupes } \\
\text { de travail }\end{array}$ & 0,778 & 0,695 \\
\hline \multirow[t]{4}{*}{$\begin{array}{l}\text { Stratégie } \\
\text { d'accompagnement }\end{array}$} & \multirow[t]{4}{*}{0,697} & \multirow[t]{4}{*}{3,641} & $\begin{array}{l}\text { J'ai développé des rapports amicaux } \\
\text { avec les parties }\end{array}$ & 0,678 & 0,493 \\
\hline & & & $\begin{array}{l}\text { J'ai essayé de simplifier l'agenda } \\
\text { de discussions en éliminant } \\
\text { les problèmes épineux }\end{array}$ & 0,670 & 0,500 \\
\hline & & & $\begin{array}{l}\text { J'ai mis en place un agenda de discussion } \\
\text { où les sujets généraux étaient d'abord traités } \\
\text { avant d'aborder des points plus spécifiques }\end{array}$ & 0,720 & 0,553 \\
\hline & & & $\begin{array}{l}\text { J'ai essayé de développer un climat } \\
\text { de confiance entre les parties }\end{array}$ & 0,777 & 0,610 \\
\hline
\end{tabular}




\begin{tabular}{|c|c|c|c|c|c|}
\hline \multicolumn{6}{|l|}{ TABLEAU 1 (suite) } \\
\hline Facteur & $\alpha$ & $\begin{array}{l}\text { Valeur } \\
\text { propre }\end{array}$ & Item & $\lambda$ & $\begin{array}{l}\text { Commu- } \\
\text { nauté }\end{array}$ \\
\hline \multirow[t]{5}{*}{$\begin{array}{l}\text { Stratégie } \\
\text { interventionniste }\end{array}$} & \multirow[t]{5}{*}{0,793} & \multirow[t]{5}{*}{3,641} & $\begin{array}{l}\text { J'ai pressé durement les parties pour } \\
\text { qu'elles arrivent à un accord }\end{array}$ & 0,715 & 0,530 \\
\hline & & & Je les ai fait changer de positions & 0,713 & 0,526 \\
\hline & & & J'ai changé leurs attentes & 0,679 & 0,508 \\
\hline & & & $\begin{array}{l}\text { Je leur ai dit que la direction qu'ils } \\
\text { souhaitaient prendre n'était pas bonne } \\
\text { pour arriver à un accord }\end{array}$ & 0,694 & 0,619 \\
\hline & & & J'ai clarifié les attentes de l'autre partie & 0,770 & 0,603 \\
\hline \multirow[t]{4}{*}{ Formation } & \multirow[t]{4}{*}{0,875} & \multirow[t]{4}{*}{2,922} & $\begin{array}{l}\text { La formation suivie sur le rôle de PCM } \\
\text { a influencé ma pratique de PCM }\end{array}$ & 0,864 & 0,746 \\
\hline & & & $\begin{array}{l}\text { Le stage sur le rôle de PCM m'a amené } \\
\text { à envisager mon rôle de PCM différemment }\end{array}$ & 0,739 & 0,546 \\
\hline & & & $\begin{array}{l}\text { Ce que j'ai appris lors de la session } \\
\text { de formation sur le rôle de PCM m'a permis } \\
\text { d'être plus efficace lors de la conciliation } \\
\text { que j'ai gérée }\end{array}$ & 0,897 & 0,804 \\
\hline & & & $\begin{array}{l}\text { La formation suivie sur le rôle de PCM } \\
\text { m'a permis de mieux gérer les demandes } \\
\text { des parties }\end{array}$ & 0,909 & 0,826 \\
\hline
\end{tabular}

Le Tableau 2 présente les statistiques descriptives des variables du modèle, ainsi que leurs corrélations.

Pour tester les hypothèses de notre modèle, nous avons mobilisé des régressions linéaires simples et multiples. Les hypothèses d'homoscédascticité et de normalité de la distribution du terme d'erreur ont été réalisées à l'aide d'une analyse des résidus. L'absence de colinéarité a été vérifiée (VIF, Tolérance). L'âge, le genre, le nombre de CMP et le rythme de la conciliation ont été considérés comme des variables de contrôle. Les résultats des différentes régressions sont présentés dans le Tableau 3.

Comme nous pouvons le voir dans le Tableau 3, les variables " contact», «tiers expert» et «tiers autorité » ont un impact positif et significatif sur la stratégie interventionniste des PCMP. Le $\mathrm{R}^{2}$ de 0,41 traduit un impact fort de ces variables sur l'orientation des PCMP vers I'action. Les variables «compétences » et «suivi » influencent positivement la mise en œuvre de la stratégie d'accompagnement. Le $R^{2}$ de 0,48 nous indique que leur pourvoir explicatif est élevé. Contrairement à ce que proposait l'hypothèse 4 , la formation suivie par le PCMP n'impacte pas positivement la mise en œuvre d'une stratégie d'accompagnement. Concernant l'impact des stratégies interventionnistes et d'accompagnement, les résultats 


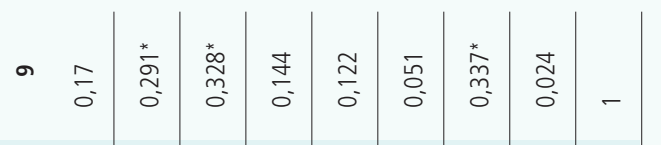

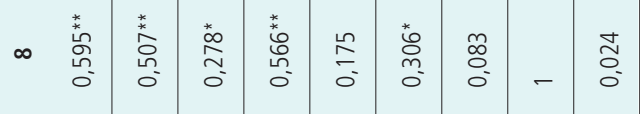

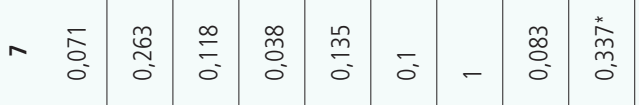

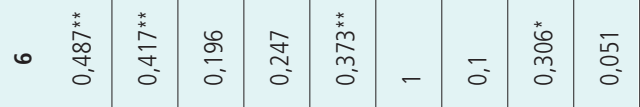

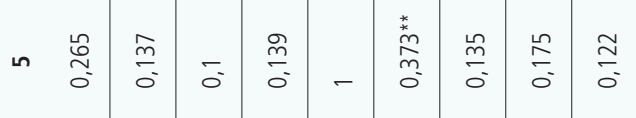

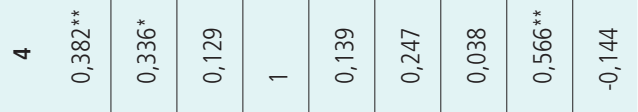

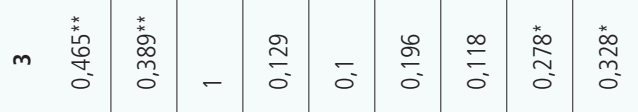

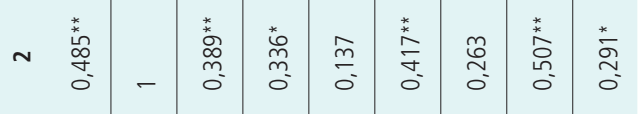

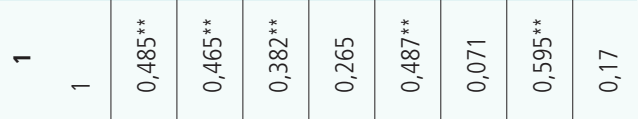

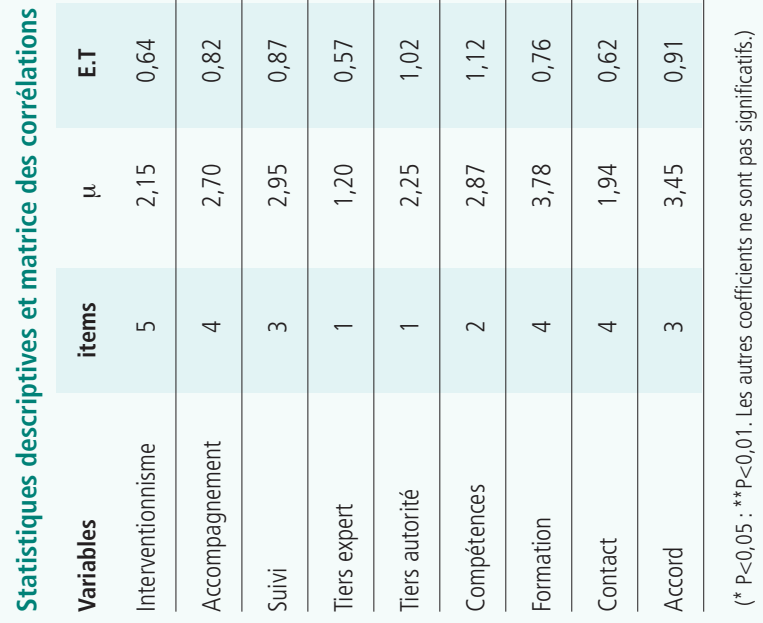




\section{TABLEAU 3}

Résultats des analyses de régression

\begin{tabular}{|c|c|c|c|c|c|c|c|}
\hline \multirow{3}{*}{$\begin{array}{l}\text { Variables } \\
\text { indépendantes }\end{array}$} & \multirow{3}{*}{$\begin{array}{l}\text { Variables }^{1} \\
\text { Variables } \\
\text { dépendantes }\end{array}$} & \multirow{2}{*}{\multicolumn{2}{|c|}{ Test $\mathbf{F}$}} & \multirow{3}{*}{$\begin{array}{l}\text { Coefficient de } \\
\text { régression }\end{array}$} & \multirow{3}{*}{ Test t } & \multirow[b]{3}{*}{ Sig } & \multirow[b]{3}{*}{$\mathrm{R}^{2}$} \\
\hline & & & & & & & \\
\hline & & $F$ & Sig & & & & \\
\hline Constante & \multirow{8}{*}{$\begin{array}{c}\text { Stratégie } \\
\text { interventionniste }\end{array}$} & \multirow{8}{*}{3,890} & \multirow{8}{*}{, 003} & 1,069 & 1,806 & 0,079 & \multirow{8}{*}{0,411} \\
\hline Contact & & & & 0,329 & 3,330 & 0,002 & \\
\hline Tiers expert & & & & 0,348 & 2,299 & 0,027 & \\
\hline Tiers autorité & & & & 0,173 & 2,147 & 0,038 & \\
\hline Âge & & & & $-0,003$ & $-0,313$ & 0,756 & \\
\hline Nombre cmp & & & & $-0,001$ & $-0,044$ & 0,965 & \\
\hline Rythme & & & & 0,044 & 0,406 & 0,687 & \\
\hline Genre & & & & $-0,203$ & $-1,503$ & 0,262 & \\
\hline Constante & \multirow{8}{*}{$\begin{array}{c}\text { Stratégie } \\
\text { d'accompagnement }\end{array}$} & \multirow{8}{*}{5,213} & \multirow{8}{*}{$<, 001 a$} & $-1,195$ & $-0,722$ & 0,141 & \multirow{8}{*}{0,483} \\
\hline Compétences & & & & 0,228 & 2,396 & 0,021 & \\
\hline Formation & & & & 0,0201 & 1,522 & 0,136 & \\
\hline Suivi & & & & 0,510 & 2,599 & 0,013 & \\
\hline Genre & & & & 0,322 & 1,493 & 0,143 & \\
\hline Âge & & & & 0,021 & 1,942 & 0,059 & \\
\hline Rythme & & & & 0,052 & 0,386 & 0,702 & \\
\hline Nombre cmp & & & & $-0,031$ & $-1,087$ & 0,284 & \\
\hline Constante & \multirow{3}{*}{ Accord } & \multirow{3}{*}{4,532} & \multirow{3}{*}{0,038} & 2,581 & 6,050 & 0,000 & \multirow{3}{*}{0,085} \\
\hline Accompagnement & & & & 0,322 & 2,129 & 0,038 & \\
\hline Interventionnisme & & & & 0,054 & 0,243 & 0,809 & \\
\hline Constante & \multirow{2}{*}{$\begin{array}{c}\text { Stratégie } \\
\text { d'accompagnement }\end{array}$} & \multirow{2}{*}{13,697} & \multirow{2}{*}{0,001} & 1,237 & 3,036 & 0,004 & \multirow{2}{*}{0,218} \\
\hline $\begin{array}{l}\text { Stratégie } \\
\text { interventionniste }\end{array}$ & & & & 0,583 & 3,701 & 0,001 & \\
\hline
\end{tabular}

1 Les hypothèses de normalité d'homoscédasticité des résidus, de non colinéarité entres les variables indépendantes ont été vérifiées.

sont nuancés. En effet, nous pouvons noter que seule la stratégie d'accompagnement a un effet positif et significatif sur l'obtention d'un accord, même si le $\mathrm{R}^{2}$ demeure modeste $(0,085)$. Il semblerait donc, à un premier niveau d'analyse, que la stratégie interventionniste ne conduise pas au succès de la médiation.

Toutefois, comme nous pouvons le voir sur le tableau ci-avant, cela ne signifie pas qu'elle n'ait pas d'effet sur la nature du résultat. En effet, la relation positive et relativement forte $\left(R^{2}=0,218\right)$ de la stratégie interventionniste sur la mise en 
œuvre de la stratégie d'accompagnement montre un effet indirect de la première sur l'accord final (via l'accompagnement), ce qui implique non seulement que les deux stratégies ne soient pas indépendantes l'une de l'autre, mais également que l'action directe sur la médiation permette d'aider à la mise en œuvre de la stratégie d'accompagnement de la médiation, qui elle-même impacte positivement le résultat de la médiation. De manière synthétique, les hypothèses $\mathrm{H} 2$ et $\mathrm{H} 4$ ne sont pas validées. $\mathrm{H1}$, H3, H5, H6a et b, H7a et b sont validées.

\section{Discussion}

\section{Apports théoriques de la recherche}

Cette recherche étudie le rôle du médiateur dans la médiation. Le premier apport de notre recherche concerne la dimensionnalité des stratégies de médiation. Nos résultats rejoignent Wall et Dunne (2012), tout comme Kolb (1981), qui proposent l'existence de deux orientations générales: I'interventionnisme et I'accompagnement, contrairement à Kressel et Pruitt (1989) et Shapiro, Drieghe et Brett (1985) qui identifient quatre profils. De plus, notre recherche permet d'identifier les stratégies développées par le médiateur et leur impact sur la survenance d'un accord, ainsi que leurs antécédents. Nos résultats confirment, dans le contexte français, l'efficacité de la stratégie d'accompagnement qui regroupe les stratégies contextuelles et réflexives (Carnavale et Pruitt, 1992). L'action sur l'agenda et la création d'un climat de confiance ont un impact positif sur l'efficacité du médiateur, ce qui démontre, en accord avec Martinez-Pecino et al. (2008) que, dans un contexte où le médiateur a dès le démarrage un mandat explicite pour agir, les tactiques contextuelles, mais aussi réflexives, sont efficaces.

Le second apport est de montrer que la stratégie interventionniste n'a pas d'impact direct sur l'accord. Cette inefficacité confirme tant les informations fournies par les différents rapports parlementaires que les travaux académiques (Grima, 2005; Le Flanchec et Rojot, 2009) soulignant que les partenaires sociaux sont soucieux de conserver la maîtrise du dialogue social. Ce résultat relativise les travaux de Posthuma (2000), Posthuma, Dworkin et Swift (2002), et Kochan et Jick (1978) soulignant l'efficacité de l'intervention du médiateur. Cependant, nos résultats font émerger une influence indirecte des stratégies interventionnistes sur l'accord par l'intermédiaire des stratégies d'accompagnement (contextuelles et réflexives). Loin de restreindre son action à un seul registre stratégique, le médiateur peut articuler l'accompagnement et l'intervention. Plus encore, il apparaît que la stratégie interventionniste ne s'oppose pas toujours, et qu'elle puisse être même menée en anticipation de la phase d'accompagnement. Cette mixité des stratégies fait apparaître le médiateur comme un stratège qui, soucieux 
d'arriver à un accord, peut mêler les diverses stratégies selon ses perceptions du contexte de la médiation. Ce résultat confirme le propos de Rothman (2014) pour qui il est impossible que le médiateur n'intervienne pas. Ce résultat étend la validité externe des recherches réalisées dans un contexte chinois (Wang, 2015) qui montrent que les médiateurs mobilisent les deux approches.

Le troisième apport de la recherche concerne les antécédents des stratégies de médiation. Notre travail nuance les résultats des recherches menées en contextes anglo-saxons et asiatiques en mettant en évidence un lien positif entre formation et stratégie d'accompagnement (McGillicuddy et al., 1987, Wall et Blum, 1991). L'absence de lien entre la formation des médiateurs et les stratégies d'accompagnement déployées est surprenante. II est possible que la nature étatique des formations suivies et du mandat fourni aux médiateurs en ait quelque peu limité les effets. Également, notre travail confirme le rôle tenu par la compétence des parties dans les orientations stratégiques du médiateur (Wall, Stark et Rhetta, 2001; Wall et Lynn, 1993; Grima, 2005). II apparaît que la perception par ce dernier d'un déficit de compétences le conduise à développer des stratégies d'accompagnement. Nous avons également testé la présence d'un tiers à la médiation, souvent peu explorée (Wall et Dunne, 2012; Wall, Stark et Standifer, 2001). Nous établissons que, quelle que soit la nature de ce tiers, d'expertise ou d'autorité, elle influence les choix stratégiques du médiateur en l'incitant à opter pour une stratégie interventionniste, confirmant les précédents travaux sur la présence d'un tiers (Bonta, 1996; Pinkley, Griffith et Northcraft, 1995), tout en précisant que cette relation est positive quelle que soit la nature de l'influence portée par le tiers: influence ou autorité.

Enfin, notre travail réalise un réel apport sur l'impact de l'activité du médiateur en dehors de la salle de médiation. Nous montrons que cette activité affecte les stratégies retenues en salle de médiation, et établissons ainsi un lien direct entre travail en salle de médiation et en dehors alors que jusqu'ici cette relation n'avait pas été posée, ou alors seulement de façon indirecte (Maoz et Terris, 2006). Plus encore, en liant précisément des activités de contact avec la stratégie interventionniste et celles de suivi avec la stratégie d'accompagnement, cette recherche valide l'existence d'une logique stratégique globale et cohérente du médiateur (Mareschal, 2005).

\section{Implications managériales de la recherche}

D'abord, il convient de rappeler le caractère exploratoire de cette recherche sur les PCMP, qui, à notre connaissance, n'ont fait l'objet d'aucun travail académique. Le taux de retour très élevé de notre questionnaire rapproche ce travail d'un recensement. Le constat dressé par Grima (2005) sur le profil des médiateurs dans les branches professionnelles demeure vrai. Les PCMP restent des fonctionnaires qui 
travaillent à titre principal, pour la plupart, au sein du Ministère du Travail. Le rôle de médiateur est donc une activité secondaire. II apparaît donc que la médiation en France dans les branches professionnelles repose sur le volontariat. Ce premier constat peut amener à se questionner sur les intentions de l'État quant à son rôle dans le dialogue social en France. L'État conduit les médiateurs à ne pouvoir jouer qu'un rôle d'accompagnateur du dialogue social, alors qu'une volonté d'intervention supposerait des moyens logistiques et humains beaucoup plus importants que ceux actuellement déployés. Ce faisant, ce résultat conduit à nuancer les discours idéologiques ambiants décrivant un État, en France, très présent dans le dialogue social. Ici, dans des situations complexes où le dialogue social est en panne, l'État ne cherche pas à s'imposer comme un troisième acteur, mais il apparaît, au contraire, comme un facilitateur promouvant une stratégie d'accompagnement plutôt qu'interventionniste. Enfin, nos résultats témoignent que la présence affirmée de l'État au travers de la présence physique de ces représentants (tiers d'expertise ou d'autorité) agit sur les choix stratégiques du médiateur. II convient donc de laisser la médiation à l'action du médiateur et des parties si l'on souhaite aboutir à un accord et éviter les positions trop affirmées du médiateur pour arriver à un accord.

\section{Conclusion}

\section{Limites de la recherche}

La nature exploratoire de ce travail, combinée à la rareté de la population d'enquête, engendre plusieurs limites qui doivent conduire à considérer les résultats proposés avec prudence. Méthodologiquement, si le taux de retour à notre questionnaire est particulièrement élevé (plus de $90 \%$ de la population-mère), plusieurs PCMP ne I'ont rempli que partiellement, ce qui nous a conduit à éliminer un nombre important de questionnaires (12). La population-mère demeure limitée à 68 personnes et plusieurs variables importantes de notre recherche, tels I'accord entre les parties ou leur niveau de formation, ne sont qu'un jugement du médiateur. De ce fait, nous ne pouvons exclure l'existence d'un biais de désirabilité sociale. Ce dernier pourrait refléter une rationalisation a posteriori des PCMP sur leurs pratiques de médiation.

La spécificité de cette population et le fait qu'il s'agisse d'un quasi recensement augmente la validité interne de cette recherche au dépend de sa validité externe, ainsi que du caractère généralisable des résultats. Du point de vue de la mesure, nous avons mobilisé l'échelle de Lim et Carnevale (1990) qui propose trois dimensions (contextuelles, substantives et réflexives), alors que notre travail fondé sur les travaux de référence du domaine n'en propose que deux (accompagnement et interventionniste). Sur ce point, nous avons conscience que les échelles utilisées pour les deux stratégies d'accompagnement et d'intervention ne rendent pas compte 
de la riche sémantique de ces deux orientations. II serait opportun de l'enrichir de tâches essentielles à la dynamique d'accord, comme structurer les échanges pour éviter l'escalade, poser des questions ouvertes, etc. II serait judicieux d'élaborer une mesure qui reflète mieux notre conception théorique et qui, pour l'instant, n'existe pas. Enfin, nous avons dû créer, pour cette recherche, la mesure de plusieurs variables. Empiriquement, il apparaît que seule une part modérée de la variance de notre variable finale, l'accord entre les parties, est expliquée par notre modèle de recherche. Ici, le contexte et l'objet de la négociation doivent être réintroduits.

\section{Pistes de recherches}

Plusieurs pistes de recherche nous apparaissent comme prometteuses. La compréhension de la dynamique de l'accord pour le médiateur ne dépend pas uniquement de ses stratégies mais d'autres éléments contextuels qu'il convient d'explorer. L'absence de liens entre formations suivies par les PCMP et stratégies qu'ils déploient mérite de plus amples investigations. II serait intéressant de se questionner sur la manière dont les PCMP s'approprient les formations suivies afin de les mettre en œuvre dans leur pratique de médiation. Replacer le médiateur au centre de la compréhension de la médiation nous semble porteur, notamment en intégrant l'influence de variables personnelles, telles que la personnalité du médiateur ou, encore, son expérience de la gestion de conflit dans d'autres contextes. Enfin, plus globalement, il conviendrait de reprendre cette recherche en redéfinissant sa variable terminale: I'accord.

La réussite d'une médiation ne peut, en effet, être limitée à la seule rédaction d'un accord (Henderson, 1996). Aborder la médiation comme un processus en plusieurs phases (Stimec et Poitras, 2009) peut également apparaître pertinent. En effet, la dynamique de l'atteinte d'un accord et le modèle de médiation transformationnelle (Bush et Floger, 2005; Lewis et Umbreit, 2015) ouvrent des perspectives de recherches intéressantes dans lesquelles l'intégration des émotions et leur régulation pourront être prises en compte.

\section{Note}

1 Selon les fascicules La Commission mixte: une procédure d'aide à la négociation de branche et Le rôle du Président de Commission mixte paritaire.

\section{Bibliographie}

Barsky, Morna (1983) «Emotional Needs and Dysfunctional Communication as Blocks to Mediation », Mediation Quarterly, 2, 55-66.

Bercovitch, Jacob, J. Theodore Anagnoson et Donnette L. Willie (1991) «Some Conceptual Issues and Empirical Trends in the Study of Successful Mediation in International Relations », Journal of Peace Research, 28, 7-17.

Bonta, Bruce D. (1996) «Conflict Resolution among Peaceful Societies: The Culture of Peacefulness », Journal of Peace Research, 33, 403-420. 
Bush, Robert A. et Baruch J. Folger (2005) The Promise of Mediation: The Transformative Approach to Conflict, San Francisco, CA: Jossey-Bass.

Carnevale, Peter J. et Dean G. Pruitt (1992) «Negotiation and Mediation», Annual Review of Psychology, 43, 531-582.

Churchill, Gilbert A. (1979) «A Paradigm for Developing Better Measures of Marketing Constructs», Journal of Marketing Research, 16, 64-73.

Girdner, Linda K. (1986) «Family Mediation: Toward a Synthesis», Conflict Resolution Quarterly, 13, 21-29.

Grima, François (2005) «Stratégies et tactiques de médiation dans un contexte français: I'exemple des présidents de Commissions mixtes », Revue de gestion des ressources humaines, 56, 49-62.

Grima, François (2006) «La médiation dans les relations professionnelles françaises: une relation à trois? », Annales des mines: Gérer et Comprendre, mars, 12-21.

Henderson, Douglas A. (1996) «Mediation Success: An Empirical Analysis», Ohio State Journal on Dispute Resolution, 11 (1), 105-148.

Hiltrop, Jean-Marie (1985) « Mediator Behavior and the Settlement of Collective Bargaining Disputes in Britain », Journal of Social Issues, 41 (2), 83-99.

Igalens, Jacques et Patrice Roussel (1998), Méthodes de recherche en gestion des ressources humaines, Paris: Economica, 203 pages.

Kochan, Thomas A. et Todd Jick (1978) «The Public Sector Mediation Process: A Theory and Empirical Examination », Journal of Conflict Resolution, 22 (2), 209-240.

Kolb, Deborah M. (1981) «Roles Mediators Play: State and Federal Practice», Industrial Relations: A Journal of Economy and Society, 20, 1-17.

Kolb, Deborah M. (1994) When Talk Works: Profiles of Mediators, San Francisco, Jossey-Bass.

Kressel, Kenneth et Dean G. Pruitt (1985) "Themes in the Mediation of Social Conflict », Journal of Social Issues, 41, 179-198.

Kressel, Kenneth et Dean G. Pruitt (1989) «A Research Perspective on the Mediation of Social Conflict», dans Mediation Research: The Process and Effectiveness of Thirdparty Intervention, San Francisco: Jossey-Bass, 394-435.

Leblay, Hervé (2003) « Médiation et conciliation au Québec: quelles possibilités d'utilisations françaises», Bilan de la mission effectuée par Hervé Leblay, directeur-adjoint du Travail, dans le cadre du programme d'échange de fonctionnaires franco-québécois, au sein de la Direction générale des relations du travail (Ministère du Travail du Québec), d'avril 1999 à mars 2001, Montréal, avril 2001.

Le Flanchec, Alice et Jacques Rojot (2009) « La médiation dans les relations du travail», Négociations, 2, 155-170.

Lewis, Ted et Mark Umbreit (2015) «A Humanistic Approach to Mediation and Dialogue: An Evolving Transformative Practice", Conflict Resolution Quarterly, 33, 3-17.

Lim, Rodney G. et Peter J. Carnevale (1990) «Contingencies in the Mediation of Disputes », Journal of Personality and Social Psychology, 58 (2), 259-272.

Maoz, Zeev et Lesley G. Terris (2006) «Credibility and Strategy in International Mediation », International Interactions, 32 (4), 409-440.

Mareschal, Patrice M. (2005) «What Makes Mediation Work? Mediators Perspectives on Resolving Disputes», Industrial Relations, 44 (3), 509-517.

Martinez-Pecino, Roberto, Lourdes Munduate, Francisco Medina et Martin Euwema (2008) «Effectiveness of Mediation Strategies in Collective Bargaining », Industrial Relations, 47 (3), 480-495.

McGillicuddy, Neil, Gary Welton et Dean Pruitt (1987) «Third-Party Intervention: A Field Experiment Comparing Three Different Models», Journal of Personality and Social Psychology, 53 (1), 104112. 
Medina, Francisco, Viriginia Vilches, Marina Otero et Lourdes Munduate (2014) «How Negotiators are Transformed into Mediators: Labor Conflict Mediation in Andalusia », Journal of Work and Organizational Psychology, 30 (3), 133-140.

Pinkley, Robin L., Terri L. Griffith et Gregory B. Northcraft (1995) «Fixed Pie à la mode: Information Availability, Information Processing, and the Negotiation of Suboptimal Agreements», Organizational Behavior and Human Decision Processes, 62 (1), 101-112.

Poitras, Jean (2013) "The Strategic Use of Caucus to Facilitate Parties' Trust in Mediators», International Journal of Conflict Management, 24 (1), 23-39.

Posthuma, Richard A. (2000) «Mediator Effectiveness: The Negotiator's Perspective», Journal of Alternative Dispute Resolution in Employment, 31 (1), 59-63.

Posthuma, Richard A., James B. Dworkin et Maris Stella Swift (2002) « Mediator Tactics and Sources of Conflict: Facilitating and Inhibiting Effects », Industrial Relations, 41 (1), 94-109.

Raiffa, Howard (1983) « Mediation of Conflicts», American Behavioral Scientist, 27, 195-210.

Richard, Arnaud et Jean-Marc Germain (2016), Rapport sur le paritarisme, Rapport №3829 déposé à I'Assemblée Nationale le 16 juin.

Ross, William H. et Carole Wieland (1996) «Effects of Interpersonal Trust and Time Pressure on Managerial Mediation Strategy in a Stimulated Organizational Dispute », Journal of Applied Psychology, 81 (2), 228-248.

Rothman, Jay (2014) «The Reflexive Mediator», Negotiation Journal, 30, 441-453.

Roussel, Patrice (2005) «Méthodes de développement d'échelles pour questionnaires d'enquête», Méthodes et Recherches, Louvain-la-Neuve: De Boeck Supérieur, 245-276.

Salzer, Jacques et Jean-Pierre Vouche (2003) «La médiation dans les organisations (entreprises, administrations, associations) », dans Jean-Pierre Bonafé-Schmitt, Jocelyne Dahan, Jacques Salzer, Marianne Souquet, Jean-Pierre Vouche, Les médiations, la médiation, Paris: ERES, 151-219.

Shapiro, Debra, Rita Drieghe et Jeanne Brett (1985) « Mediator Behavior and the Outcome of Mediation », Journal of Social Issues, 41, 101-114.

Stimec, Arnaud et Sylvie Adijès (2015) La médiation en entreprise, Paris: Dunod.

Stimec, Arnaud et Jean Poitras (2009) «Building Trust with Parties: Are Mediators Overdoing it?», Conflict Resolution Quarterly, 26 (3), 317-331.

Susskind, Lawrence (1981) «Environmental Mediation and the Accountability Problem», Vermont Law Review, 6, 1-47.

Touzard, Hubert (1977) La médiation et la résolution des conflits, Paris: PUF.

Tyler, Tom R. (1987) «The Psychology of Disputant Concerns in Mediation», Negotiation Journal, 3, 367-374.

Wall, James A. et Michael Blum (1991) «Community Mediation in the People's Republic of China», Journal of Conflict Resolution, 35 (1), 3-20.

Wall, James A. et Suzanne Chan-Serafin (2009) «Processes in Civil Case Mediation », Conflict Resolution Quarterly, 26 (3), 261-291.

Wall, James A. et Timothy C. Dunne (2012) «Mediation Research: A Current Review», Negotiation Journal, 28 (2), 217-244.

Wall, James A. et Ann Lynn (1993) «Mediation: A Current Review», Journal of Conflict Resolution, 37 (1), 160-194.

Wall, James A., John B. Stark et Rhetta L. Standifer (2001) « Mediation: A Current Review and Theory Development », Journal of Conflict Resolution, 45 (3), 370-391.

Wang, Jian (2015) «Neutral, Biased, or Both? Discursive Construction of a Mediator's Dual Role», Negotiation Journal, 31 (1), 47-65. 
Welton, Gary L., Dean G. Pruitt, Neil B. McGillicuddy, Carol A. Ippolito et Jo M. Zubek (1992) «Antecedents and Characteristics of Caucusing in Community Mediation», International Journal of Conflict Management, 3 (4), 303-317.

Zartman, William I. et Saadia Touval (1985) «International Mediation: Conflict Resolution and Power Politics », Journal of Social Issues, 41 (2), 27-45.

\section{RÉSUMÉ}

\section{Antécédents et efficacité des stratégies de médiation: l'intervention des présidents de Commissions mixtes paritaires en France}

Le but de cet article est d'identifier les stratégies développées par le médiateur, ainsi que leurs antécédents. S'appuyant sur une enquête quantitative réalisée auprès de 51 présidents de Commission mixte paritaire (PCMP) ayant un rôle de médiateur au niveau des branches professionnelles, cette recherche établit deux résultats.

Premièrement, elle distingue deux stratégies développées par le médiateur: la stratégie d'accompagnement et la stratégie interventionniste. La première permet au médiateur d'amener les parties au conflit à l'accord. La seconde stratégie n'a pas d'effets directs sur la résolution de l'accord. Cependant, nos résultats démontrent l'existence d'un effet indirect de cette stratégie interventionniste. Celle-ci est efficace lorsqu'elle est associée à la stratégie d'accompagnement.

Deuxièmement, l'intervention d'un tiers expert ou d'autorité, soit un suivi de l'activité des parties en dehors de la salle de médiation, facilite la stratégie interventionniste alors que la compétence des parties, notamment un contact régulier avec ces dernières, est lié à la stratégie d'accompagnement. Dépassant les approches descriptives, ce travail propose une meilleure compréhension du comportement du médiateur, une intervention dynamique et complexe lors du processus de médiation.

Cette recherche apparait comme un nouveau point de départ pour comprendre les dynamiques de médiations dans un contexte où le rôle des partenaires sociaux dans la définition de la réglementation sociale s'affirme. Les auteurs suggèrent d'intégrer dans l'analyse des dynamiques de médiation, les facteurs contextuels et personnels liés aux différents acteurs que sont le médiateur, les parties prenantes, ainsi que les tiers extérieurs.

Les limites de ce travail demeurent la taille de son échantillon, ainsi que l'absence de triangulation des sources qui auraient permis d'obtenir une vision plus éclairée de l'action du médiateur. De plus, l'étude ayant été conduite en France, elle n'exclut pas l'existence de biais culturels. C'est pourquoi ce travail ouvre des perspectives multiples de recherches sur le sujet du rôle du médiateur dans les dynamiques de médiations.

MOTS-CLÉs: médiateur, médiation, stratégie, relations sociales. 


\section{SUMMARY}

\section{Antecedents and Effectiveness of Mediation Strategies: The Intervention of Presidents of Joint Committees in France}

The purpose of this article is to identify strategies developed by mediators, as well as their antecedents. Based on a quantitative survey conducted with 51 Joint Committee Presidents acting as mediators at the professional branch level, this research brings two main results.

Firstly, it distinguishes between two strategies developed by the mediator: the support strategy and the interventionist strategy. The former allows the mediator to bring parties in conflict to agreement. Conversely, the latter has no direct effect on the resolution of the agreement. However, our results show the existence of an indirect effect of an interventionist strategy. Furthermore, this is shown to be effective when it is associated with a support strategy.

Secondly, the intervention of a third-party expert or authority, as a follow-up to the parties' activities outside the mediation room, facilitates the interventionist strategy, while the parties' competence, especially when there is regular contact with these parties, is linked to the support strategy. Going beyond descriptive approaches, this work promotes a better understanding of the mediator's behaviour, reflecting dynamic and complex interventions during the mediation process.

This research reflects a new starting point in understanding the dynamics of mediation in a context where the role of the social parties defining social regulation is strengthened. The authors suggest integrating into the analysis of mediation dynamics contextual and personal factors linked to the different actors, such as the mediator, stakeholders, and external third parties.

The limits of this research lie in the size of its sample, as well as the absence of a triangulation of sources that would have allowed the authors to obtain a clearer vision of the mediator's action. Moreover, this study was conducted in France and therefore the possible existence of cultural bias cannot be excluded.

This work, however, opens up multiple perspectives for research on the subject of the role of the mediator in the dynamic context of mediation.

KEYWORDS: mediator, mediation, strategy, social relations.

\section{RESUMEN}

\section{Antecedentes y eficacidad de las estrategias de mediación: la intervención de los presidentes de Comisiones mixtas paritarias en Francia}

El objetivo de este artículo es de identificar las estrategias desarrolladas por el mediador, así como sus antecedentes. Basada en una encuesta cuantitativa realizada con 51 presidentes de Comisión mixta paritaria (CMP) que tienen un 
rol mediador a nivel de las ramas profesionales, esta investigación establece dos resultados.

En primer lugar, se distinguen dos estrategias desarrolladas por el mediador: la estrategia de acompañamiento y la estrategia intervencionista. La primera permite al mediador de conducir las partes en conflicto hacia un acuerdo. La segunda estrategia no tiene efectos directos en la resolución del acuerdo. Sin embargo, nuestros resultados muestran la existencia de un efecto indirecto de esta estrategia intervencionista. Esta es eficaz cuando está asociada a la estrategia de acompañamiento.

En segundo lugar, la intervención de un tercero (experto o autoridad), es decir un seguimiento de la actividad de las partes fuera de la sala de mediación, facilita la estrategia intervencionista mientras que la competencia de las partes, especialmente un contacto regular con estas últimas, está vinculada a la estrategia de acompañamiento. Más allá de los enfoques descriptivos, este trabajo propone una mejor comprensión del comportamiento del mediador, una intervención dinámica y compleja durante el proceso de mediación.

Esta investigación marca un punto decisivo para comprender las dinámicas de las mediaciones en un contexto donde el rol de los actores sociales en la definición de la reglamentación social se afirma. Los autores sugieren de integrar en el análisis las dinámicas de mediación, los factores contextuales y personales vinculados a los diferentes actores que son el mediador, las partes involucradas, así como los terceros exteriores.

Los límites de este trabajo residen en la talla de la muestra y la ausencia de triangulación de las fuentes que hubiera permitido obtener una visión más clara de la acción del mediador. Además, el estudio conducido en Francia, no excluye la existencia de sesgos culturales. Por ello, este trabajo abre las perspectivas a múltiples investigaciones sobre el sujeto del rol del mediador en las dinámicas de mediaciones.

PALABRAS CLAVES: mediador, mediación, estrategia, relaciones sociales. 